\title{
Der Mythos von Isis und Osiris als Ursprung der Ringparabel?
}

Das Fragezeichen im Titel meines Beitrags ist nur allzu berechtigt. Ob wirklich eine Überlieferungslinie von einer Episode des ägyptischen Osiris-Mythos zu den Ursprüngen der Ringparabel führt, ist eine offene Frage. Die mögliche Parallele scheint aber eng genug, um sie sich genauer anzuschauen.

Die entscheidende Passage des ägyptischen Mythos steht in der Bibliotheca Historica des Diodor von Sizilien, eines Zeitgenossen von Cicero, der im 1. Jahrhundert v. Chr. schrieb, sich für seinen Ägyptenbericht jedoch weitestgehend auf einen älteren Autor, Hekataios von Abdera, stützte. Dieser war Ende des 4. Jahrhunderts nach Alexandria gezogen, um Ptolemaios I. beim Aufbau des Musaions und der Bibliothek von Alexandrien zu helfen. Er schrieb eine heute verlorene mehrbändige Geschichte Ägyptens, die Ptolemaios für die Regierung des Landes als Orientierung dienen sollte. So wird auch Diodors Version des Osiris-Mythos auf Hekataios zurückgehen:

Obwohl die Priester seit ältesten Zeiten den Tod des Osiris als strengstes Geheimnis überliefert haben, ist doch im Laufe der Jahre durch einige von ihnen dieses verborgene Wissen der Menge bekannt geworden. Dies ist die Geschichte, wie sie sie erzählen: Als Osiris über Ägypten als legitimer König herrschte, wurde er von seinem Bruder Typhon, einem gewalttätigen und gottlosen Menschen, ermordet. Typhon zerteilte die Leiche in 26 Teile und gab jedem Mitglied seiner Bande einen Körperteil, weil er wollte, dass alle an der Freveltat teilhaben und dachte, dass er auf diese Weise in ihnen Mitkämpfer und Verteidiger seiner Herrschaft gewinnen würde.

Isis aber, die Schwester und Gattin des Osiris, rächte seinen Tod mit Hilfe ihres Sohnes Horus und wurde Königin von Ägypten, nachdem sie Typhon und seine Bande geschlagen hatten. [...] Isis nun sammelte alle Körperglieder des Osiris zusammen mit Ausnahme des Schamglieds; und da sie wünschte, daß das Grab ihres Gemahls geheim bliebe und doch von allen Ägyptern verehrt würde, erreichte sie ihr Ziel auf die folgende Weise. Um jedes Körperglied formte sie, wie erzählt wird, aus Wachs und Spezereien eine menschliche Figur in der Größe des Osiris. Dann rief sie die Priesterschaften zusammen und ließ sie einen Eid schwören, niemandem das Geheimnis zu verraten, das sie ihnen anvertrauen wollte. Dann nahm sie jede Gruppe einzeln beiseite und eröffnete ihr, dass sie ihnen allein das Begräbnis der Leiche übertragen wolle, und nachdem sie ihnen noch einmal die Wohltaten des Osiris in Erinnerung gerufen hatte, ermahnte sie sie, ihm göttliche Ehren zu erweisen und ihm auch einige der Tiere, die bei ihnen lebten, nach ihrer Wahl zu weihen, ihnen zu deren Lebzeiten die Ehren zu erweisen wie vormals dem Osiris und sie nach deren Tod in derselben Weise zu bestatten wie Osiris.

Und weil Isis die Priester dazu bringen wollte, diese Ehren auch aus eigenem Interesse zu erweisen, wies sie ihnen den dritten Teil des Landes zu, um die Kosten des Kults zu 
bestreiten. Und die Priester, sagt man, gedachten der Wohltaten des Osiris, eiferten, der Isis in ihren Bitten zu Gefallen zu sein und taten, auch aus eigenem Interesse, alles, was Isis vorgeschlagen hatte. Aus diesem Grund glaubt bis heute jede Priesterschaft, dass Osiris in ihrem Gau begraben sei, hält die Tiere in Ehren, die ihm ursprünglich geweiht wurden und erneuert, wenn sie sterben, in den Begräbnisriten für sie die Trauer um Osiris.

Der Kult der heiligen Stiere, die sie Apis und Mnevis nennen, wurde in ganz Ägypten eingeführt, weil diese Tiere mehr als andere denen geholfen hatten, die das Getreide entdeckt und die Aussaat und alle anderen Feldarbeiten entwickelt hatten, von denen die Menschheit profitiert. ${ }^{1}$

Dieser Bericht ist eine ätiologische Legende, die vier auffallende Eigenheiten der ägyptischen Religion erklären will: 1. Wie es angeht, dass feder ägyptische Gau sein eigenes Osiris-Grab besitzt, 2. warum Kult und Mythos des Osiris mit soviel Tabus und Geheimnissen umgeben sind, 3. was es mit dem Kult der heiligen Tiere auf sich hat und 4. wie es kommt, dass die Tempel so viel Land besitzen. Uns interessieren im Zusammenhang mit der Ringparabel natürlich nur die ersten beiden Punkte: Wie ist es zu erklären, dass alle Gaue Ägyptens sich rühmen, das wahre Osiris-Grab zu besitzen und dessen Kult mit strikter Geheimhaltung umgeben? Dieser erste Teil der Geschichte enthält wiederum zwei bedeutende Motive, denen wir zunächst einzeln nachgehen wollen:

1. die Tat des Seth-Typhon, den Leichnam des ermordeten Osiris zu zerstückeln und die einzelnen Glieder an seine Komplizen zu verteilen, um sich deren Treue und Hilfe zu versichern;

2. die Tat der Isis, diese einzelnen von ihr eingesammelten Körperteile auf die einzelnen Gaue des Landes wieder auszuteilen (anstatt sie zu einem vollständigen Körper zusammenzusetzen).

Diese beiden Motive kommen auch in einer leicht veränderten, weniger prägnanten Fassung bei Plutarch, der ungefähr 150 Jahre nach Diodor und ca. 430 Jahre nach Hekataios schrieb, in seiner Abhandlung De Iside et Osiride vor:

Typhon soll des Nachts [...] darauf [sc. auf den Sarg mit der Leiche des von ihm ermordeten Osiris] gestoßen sein. Er habe die Leiche erkannt, in vierzehn Teile zerstückelt und diese zerstreut. Als Isis das erfuhr, habe sie die Teile wieder gesucht, in einem Papyrusboot durch die Sümpfe fahrend. [...] Daher komme es auch, daß es in Ägypten viele angebliche Gräber des Osiris gibt: Isis habe, wo immer sie auf einen Körperteil stieß, ein Grab bereitet. Andere bestreiten das; Isis habe vielmehr Abbilder hergestellt und sie jeder einzelnen Stadt geschenkt, so als ob sie ihr die Leiche schenkte, damit er an mehr Stellen Ehren genieße

1 Diodorus Siculus: Bibliotheca historica I 21. In: Diodorus of Sicily. Mit einer englischen Ubersetzung von C. H. Oldfather. Bd.1: Books I and II, 1-34. London 1933 (The Loeb Classical Library 279), S. 64-69. 
und Typhon, wenn er über Horus obsiegen würde, bei der Suche nach dem wahren Grab unter den vielen, die genannt und gezeigt werden, aufgeben müßte. Der einzige Körperteil des Osiris, den Isis nicht gefunden habe, sei das Schamglied gewesen. Es sei sofort in den Fluß geworfen worden, und von ihm hätten gefressen der ägyptische Karpfen (lepidōtós), die Meeräsche (phágros) und der Nasennilhecht (oxýrhynchos) - Fische, welche von den Ägyptern aus religiöser Scheu besonders gemieden würden; Isis habe als Ersatz eine Nachbildung verfertigt und geweiht, den Phallos, dem auch heute noch die Ägypter ein Fest feierten. $^{2}$

Hier gibt es zwar das auch sonst vielfach belegte Motiv der Zerstückelung, aber nicht das Motiv der Verteilung der Körperglieder an die Verschwörer, das wohl eine Besonderheit der Diodor/Hekataios-Fassung darstellt. Dafür ist aber das Motiv der Anfertigung identischer Reproduktionen, das eine gewisse Parallele zur Ringparabel darstellt, beiden Fassungen gemeinsam.

Das Motiv der verteilten Körperglieder wiederum hat in der Bibel eine erstaunliche Parallele und zudem, zweifellos von dem biblischen Bericht genauso abhängig wie Lessings Verwendung der Ringparabel von Boccaccio, in Heinrich von Kleists Hermannsschlacht. Obwohl ich damit den engeren thematischen Rahmen der Ringparabel verlasse, möchte ich doch auch diesem Motiv des Mythos ein wenig nachgehen.

In Kapitel 19 und 20 des Buchs der Richter lesen wir:

Und siehe, da kam ein alter Mann von seiner Arbeit vom Felde am Abend, und er war auch vom Gebirge Ephraim und ein Beisasse zu Gibea; aber die Leute des Orts waren Benjaminiter. Und da er seine Augen aufhob und sah einen Fremden auf der Gasse, sprach er zu ihm: Wo willst du hin? und wo kommst du her? Er aber antwortete ihm: Wir reisen von Bethlehem-Juda, bis wir kommen an die Seite des Gebirges Ephraim, daher ich bin; und bin gen Bethlehem-Juda gezogen und ziehe jetzt zum Hause des HERRN, und niemand will mich beherbergen. Wir haben Stroh und Futter für unsre Esel und Brot und Wein für mich und deine Magd und für den Knecht, der mit deinem Diener ist, daß uns nichts gebricht. Der alte Mann sprach: Friede sei mit dir! Alles was dir mangelt findest du bei mir; bleibe nur nicht über Nacht auf der Gasse. Und führte ihn in sein Haus und gab den Eseln Futter, und sie wuschen ihre Füße und aßen und tranken.

Und da ihr Herz nun guter Dinge war, siehe, da kamen die Leute der Stadt, böse Buben, und umgaben das Haus und pochten an die Tür und sprachen zu dem alten Mann, dem Hauswirt: Bringe den Mann heraus, der in dein Haus gekommen ist, daß wir ihn erkennen. Aber der Mann, der Hauswirt, ging zu ihnen heraus und sprach zu ihnen: Nicht, meine Brüder, tut nicht so übel; nachdem dieser Mann in mein Haus gekommen ist, tut nicht eine solche Torheit! Siehe, ich habe eine Tochter, noch eine Jungfrau, und dieser ein Kebsweib;

2 Plutarch: De Iside et Osiride 18. In: Ders.: Drei religionsphilosophische Schriften. Uber den Aberglauben. Über die späte Strafe der Gottheit. Über Isis und Osiris. Griechisch-deutsch. Ubersetzt und hg. von Herwig Görgemanns unter Mitarbeit von Reinhard Feldmeier und Jan Assmann. Düsseldorf, Zürich 2003, S. $164 \mathrm{f}$. 
die will ich herausbringen. Die mögt ihr zu Schanden machen, und tut mit ihr, was euch gefällt; aber an diesen Mann tut nicht solche Torheit. Aber die Leute wollten ihm nicht gehorchen. Da faßte der Mann sein Kebsweib und brachte sie zu ihnen hinaus. Die erkannten sie und trieben ihren Mutwillen an ihr die ganze Nacht bis an den Morgen; und da die Morgenröte anbrach, ließen sie sie gehen.

Da kam das Weib hart vor morgens und fiel nieder vor der Tür am Hause des Mannes, darin ihr Herr war, und lag da, bis es licht ward. Da nun ihr Herr des Morgens aufstand und die Tür auftat am Hause und herausging, daß er seines Weges zöge, siehe, da lag sein Kebsweib vor der Tür des Hauses und ihre Hände auf der Schwelle. Er aber sprach zu ihr: Stehe auf, laß uns ziehen! Aber sie antwortete nicht. Da nahm er sie auf den Esel, machte sich auf und zog an seinen Ort. Als er nun heimkam, nahm er ein Messer und faßte sein Kebsweib und zerstückte sie mit Gebein und mit allem in zwölf Stücke und sandte sie in alle Grenzen Israels. Wer das sah, der sprach: Solches ist nicht geschehen noch gesehen, seit der Zeit die Kinder Israel aus Ägyptenland gezogen sind, bis auf diesen Tag. Nun bedenkt euch über dem, gebt Rat und sagt an!

Da zogen die Kinder Israel aus und versammelten sich zuhauf wie ein Mann, von Dan bis gen Beer-Seba und vom Lande Gilead zu dem HERRN gen Mizpa; und traten zuhauf die Obersten des ganzen Volks aller Stämme Israels in der Gemeinde Gottes, vierhunderttausend Mann zu Fuß, die das Schwert auszogen. [...] Und die Kinder Israel sprachen: Sagt, wie ist das Übel zugegangen? Da antwortete der Levit, des Weibes Mann, die erwürgt war, und sprach: Ich kam gen Gibea in Benjamin mit meinem Kebsweibe, über Nacht dazubleiben. Da machten sich wider mich auf die Bürger zu Gibea und umgaben mich im Hause des Nachts und gedachten, mich zu erwürgen; und haben mein Kebsweib geschändet, daß sie gestorben ist. Da faßte ich mein Kebsweib und zerstückte es und sandte es in alle Felder des Erbes Israels; denn sie haben einen Mutwillen und eine Torheit getan in Israel. Siehe, da seid ihr Kinder Israel alle; schafft euch Rat und tut hierzu! (Ri 19,20-20,7).

Warum zerstückelt der Mann die Leiche seiner von den Leuten zu Gibea zu Tode vergewaltigten Nebenfrau in zwölf Teile und sendet sie in alle Bezirke Israels? Um die zwölf Stämme an ihr Bündnis zu mahnen und zu gemeinsamem Handeln aufzufordern. ${ }^{3}$ Indem sie sich zur Rache vereinen, kommen die zwölf Körperteile wieder zur Einheit eines Körpers zusammen. Ein entsprechendes Motiv treibt den Seth/Typhon bei Diodor, die Leiche des Osiris in 26 Teile zu zerstückeln und jedem seiner Komplizen einen Teil zu geben. Sie sollen so zusammenhalten wie ein Körper, und sie sollen sich kollektiv mit der Mordtat identifizieren. So sollen sich auch die Stämme Israels kollektiv mit dem dem Mann aus Ephraim angetanenen Unrecht und mit seiner Rache identifizieren. Die Zerstückelung der Leiche und Verteilung der Körperteile ist ein politisches Symbol. Es symbolisiert einmal

$3 \mathrm{Zu}$ den Riten des `Heiligen Krieges` gehörte die Sitte, ein Tier zu zerstückeln und die Teile an die zwölf Stämme zu senden, um sie zur Teilnahme am Feldzug aufzufordern; vgl. 1 Sam 11,6-7. Dort verbindet sich die Sendung der Fleischstücke mit der Drohung: »Wer nicht auszieht, Saul und Samuel nach, des Rinder soll man also tun!« Damit wird an das Bündnis appelliert, welches dadurch geschlossen wird, dass die Parteien zwischen den zerteilten Tieren hindurchschreiten. 
die zu gemeinsamem politischen Handeln verschworene $>$ Körperschaft $\prec$ der Komplizen des Seth/Typhon und das andere Mal die zwölf Stämme, die gemeinsam das Volk Israel bilden.

In seinem von glühendem Nationalismus und Franzosenhass geprägten Drama Die Hermannsschlacht lässt Heinrich von Kleist Hermann, den Cherusker, dieselbe Strategie ergreifen, um die deutschen Stämme zum gemeinsamen Vorgehen gegen den römischen Feind aufzustacheln. Als er zufällig auf eine durch die Vergewaltigung eines jungen germanischen Mädchens namens Hally aufgewühlte Volksmasse trifft, schlägt er vor, Hallys Leiche in 15 Teile zu zerlegen und diese unter die 15 germanischen Stämme zu verteilen:

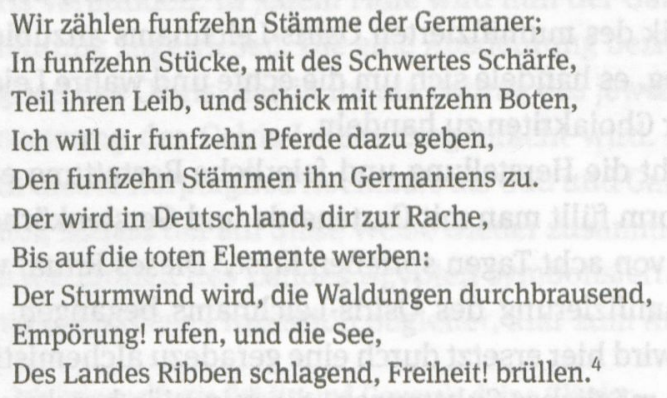

In jedem einzelnen der ersten fünf Verse dieses Ausschnitts kommt die Zahl "funfzehn" vor. Mit dieser Zahl wird die Gesamtheit der germanischen Stämme beschworen, die zu einer Einheit und zu gemeinsamem politischen Handeln verschmolzen werden sollen. In der Bibel ist zwölf die entscheidende Zahl: Sie bezeichnet die Summe der israelitischen Stämme. Was nun Ägypten betrifft, gehen die beiden Versionen des Mythos, Diodor und Plutarch, auseinander. Bei Diodor sind es 26 Körperteile, was der Anzahl an Osirisgräbern entspricht, bei Plutarch wird der Körper in 14 Teile geteilt. Beiden Autoren ist natürlich bekannt sie erwähnen es oft genug an anderen Stellen ihrer Werke -, dass Ägypten 42 Gaue zählt, 22 oberägyptische und 20 unterägyptische. Die Zahl 26 ergibt hier keinen Sinn, 14 jedoch, die zumindest dreimal in 42 enthalten ist, kann pars pro toto für die Zahl 42 stehen, denn den Leichnam des Osiris in 42 Teile zu zerstückeln kommt natürlich nicht infrage. Ebenso wichtig aber wie die Verteilung der membra disiecta des zerstückelten Osiris-Leichnams auf die $14=42$ ägyptischen Gaue ist ihre rituelle Vereinigung. So wie die zwölf israelitischen Stämme und

4 Heinrich von Kleist: Die Hermannsschlacht. In: Ders.: Sämtliche Werke und Briefe. Hg. von Helmut Sembdner. Zweibändige Ausgabe in einem Band. Bd.1. München 2001, S. 533-628, hier S. 590 f. (4. Aufz., 6. Auftr.). 
die 15 germanischen Stämme sollen sich auch die 42 ägyptischen Gaue zusammentun, um den zerstückelten Leib zu restituieren. Das geschieht in Ägypten aber nicht in der Form eines militärischen Zusammenschlusses, sondern im Rahmen eines jährlich begangenen Fests, des Choiakfests. Wir wissen nicht, wie weit diese spezifische Tradition der rituellen Gliedervereinigung in die Spätzeit zurückreicht. In den Quellen tritt sie uns erst in ptolemäischer Zeit entgegen. Die Choiakriten bilden das wichtigste, in allen Tempeln des Landes gefeierte, wahrhaft `nationale` Ritual des spätzeitlichen Ägypten.

Während nun das erste Motiv, die Zerstückeluhg der Osiris-Leiche, in den ägyptischen Quellen zahlreiche Parallelen hat und ein zentrales Element des ägyptischen Osiris-Mythos darstellt, scheint es sich beim zweiten Motiv, der List der Isis, jedem Gau eine Replik des mumifizierten Osiris-Leichnams anzubieten mit der geheimen Versicherung, es handele sich um die echte und wahre Leiche, um ein Missverständnis dieser Choiakriten zu handeln.

Im Zentrum der Riten steht die Herstellung und feierliche Bestattung einer `Kornmumier. Eine goldene Form füllt man mit Gartenerde und Gerstenkörnern, die man über einen Zeitraum von acht Tagen sprießen lässt. ${ }^{5}$ Dieses Ritual wird als Einbalsamierung und Mumifizierung des Osiris-Leichnams begangen. Der Prozess der Einbalsamierung wird hier ersetzt durch eine geradezu alchemistisch zu nennende Ansetzung aller möglicher Substanzen, die minutiös beschrieben werden, von denen die Kornmumie nur eine ist, und die alle dem Umwandlungsbzw. Sublimationsprozess der >Verklärung` unterworfen werden. Der entscheidende Aspekt dabei ist die Umwandlung aus der Zerrissenheit in die wiederhergestellte Einheit. Das wird in Form einer Kanopenprozession begangen. ${ }^{6}$ Dabei

5 Hierfür gibt es zahlreiche Belege, z. B. den Festkalender im Grab des Gottesvaters Neferhotep TT 50 wohl aus frühramessidischer Zeit (um 1300 v. Chr.); siehe hierzu Jan Assmann unter Mitarbeit von Martin Bommas und Andrea Kucharek: Altägyptische Totenliturgien. Bd. 2: Totenliturgien und Totensprüche in Grabinschriften des Neuen Reiches. Heidelberg 2005, S. 433 f., sowie Émile Chassinat: Le mystère d'Osiris au mois de Khoiak. 2 Bde. Kairo 1966 und 1968, hier Bd. 1, S. 53 ff., und Bd. 2, S. $196 \mathrm{ff}$. Acht Tage ist auch die Zeit, die ein sAdonisgärtlein ‘ braucht, um zu sprießen; vgl. Platon: Phaidros 276b.

6 Siehe dazu Horst Beinlich: Die »Osirisreliquien«. Zum Motiv der Körperzergliederung in der altägyptischen Religion. Wiesbaden 1984 (Ägyptologische Abhandlungen 42) und Laure Pantalacci: Une conception originale de la survie osirienne d'après les textes de Basse Epoque. In: Göttinger Miszellen 52 (1981), S. 57-66; dies.: Sur quelques termes d'anatomie sacrée dans les listes ptolemaiques de reliques osiriennes. In: Göttinger Miszellen 58 (1982), S. 65-72; dies.: Sur les méthodes de travail des décorateurs tentyrites. In: Le Bulletin de l'Institut français d'archéologie orientale 86 (1986), S. 267-275; dies.: Décor de la 2ème chapelle Osirienne de l'est (sud) sur le toit du temple de Dendara. In: Akten des vierten Internationalen Ägyptologen-Kongresses München 1985. Hg. von Sylvia Schoske. Bd. 3: Linguistik - Philologie - Religion. Hamburg 1989 (Studien 
treten die Personifikationen der 42 Gaue des Landes auf, geleitet von großen und übergeordneten Gottheiten, und bringen jeweils in einer Vase mit figürlichem Deckel (Kanope) ein Körperglied des erschlagenen Osiris, aus dem dann der Leib rituell wieder zusammengesetzt wird. Unter den Vorschriften zur Durchführung des Choiakfests gibt es auch genaue Anweisungen, wie die Körperglieder des Osiris herzustellen sind. Sie werden aus besonderem Teig in speziellen Holzformen gebacken. So kann man vermuten, dass die Vasen zusammen mit Nilwasser je eines dieser Körperglieder enthalten.

Immer wieder ist in den begleitenden Texten von den >Ausflüssen ‘ des Osiris die Rede. ${ }^{7}$ In der Spätzeit werden Nil und Nilüberschwemmung systematisch mit Osiris verbunden. In jedem Falle wird nun der Gabe eine doppelte sakramentale Bedeutung zugewiesen. Die eine Ausdeutung bezieht sie auf das spezifische Körperglied des Osiris, das in ihr als Beitrag des jeweiligen Gaues zur Wiederzusammensetzung des Osiris-Leibes dargebracht wird. Auf einer zweiten Ebene wird dann dieses Körperglied nochmals als Gau und Gauhauptstadt des Landes ausgedeutet, sodass der auf diese Weise wieder zusammengesetzte und beseelte OsirisLeib die Einheit des Landes Ägypten symbolisiert. Das kommt in den Reden des Königs, der diese Prozession begleitet, klar zum Ausdruck:

Ich bringe dir die Städte und Gaue als deine Glieder.

Zugewiesen werden die Götter zu deinem Leib als dein Mysterium.

Die Gottesglieder sind die Gaugötter in ihrer wahren Gestalt.

Ich bringe dir die Göttergemeinschaft Oberägyptens in ihrer Gesamtheit:

Deine Gottesglieder sind an ihrem Platz versammelt. ${ }^{8}$

Ich bringe dir die Hauptstädte der Gaue: Es sind deine Glieder, sie sind dein $\mathrm{Ka}$, der bei dir ist.

Ich bringe dir deinen Namen, deinen $\mathrm{Ba}$, deinen Schatten,

deine Gestalt, dein Bild und die Städte deiner Gaue.

zur altägyptischen Kultur, Beihefte 3), S. 327-337, sowie Jean-Claude Goyon: Momification et Recomposition du Corps Divin. Anubis et les canopes. In: Funerary Symbols and Religion. Essays dedicated to Professor M. S. H. G. Heerma van Voss on the occasion of his retirement from the Chair of the History of Ancient Religions at the University of Amsterdam. Hg. von J. H. Kamstra, H. Milde und K. Wagtendonk. Kampen 1988, S.34-44. In den Osiris-Kapellen von Dendera ist diese Prozession im mittleren Raum der westlichen Kapelle dargestellt; vgl. dazu Sylvie Cauville: Le temple de Dendara. Les chapelles osiriennes. 2Bde. Kairo 1997, hier Bd. 1, S. 40-51 (Dendara X, 71-91), und Bd. 2, S. 33-45.

7 Jeannot Kettel: Canopes, rdw.w d'Osiris et Osiris-Canope. In: Hommages à Jean Leclant. Bd. 3: Études Isiaques. Hg. von Catherine Berger, Gisèle Clerc und Nicolas Grimal. Kairo 1994 (Bibliothèque d'étude 106/3), S. 315-330.

8 Beinlich (Anm. 6), S. 89; Cauville (Anm. 6), Bd. 1, S. 40 (Dendara X, 71 f.). 
Ich bringe dir die Hauptgötter Unterägyptens zusammen vereint. Alle deine Körperglieder, sie sind vereint. ${ }^{9}$

(Rede der Meret des Nordens:)

Ich bringe dir die 42 Städte und Gaue:

Sie sind deine Glieder.

Gegründet ist für dich das ganze Land als Ort deines Körpers,

du gehst darin, du kommst darin.

Ich bringe dir die Körperglieder, damit du lebst. ${ }^{10}$

(Rede des Upuaut des Nordens:)

Ich bringe dir deine Gaue,

die zweiundvierzig sind mit dir: Sie sind dein Leib.

Deine Knochen sind für dich verknüpft, dein Name ist `Herrscher der Hauptstädte`,

das ganze Land bewahrt deine Grabstätte.

So wahr Re lebt, du ruhst Tag für Tag

in deinem Namen sDer lebt und ruht ‘. ${ }^{11}$

Im Grunde wird hier das Balsamierungsritual auf das ganze Land Ägypten appliziert, um seine Zerrissenheit $\mathrm{zu}$ heilen, um es zu vereinen, $\mathrm{zu}$ beseelen und $\mathrm{zu}$ erneuern. Die Festperiode beginnt mit der Auffindung und Einbalsamierung der 42 zerstreuten Glieder des erschlagenen Osiris, die aus den 42 Gauen des Landes herbeigebracht, rituell vereint und belebt werden, und endet mit der Beisetzung des Osiris sowie der Thronbesteigung des Horus, seines Sohnes und Rächers.

Da die Choiakriten in der Spätzeit in allen religiösen Zentren des Landes gefeiert wurden und jeweils in der Prozession zum Osiris-Grab und der Beisetzung der Kornmumie kulminierten, wird dieser Brauch bei den griechischen Zuschauern die Frage provoziert haben, wie es denn sein könne, dass Osiris so viele über ganz Ägypten verstreute Gräber haben könne. Da wird man ihnen vermutlich die Geschichte von der List der Isis erzählt haben, die in den ägyptischen Quellen nicht belegbar ist und vermutlich eine erst später ad hoc entstandene Legende darstellt.

Das Choiakfest hat seinen Ursprung im Totenkult, im Ritual der Einbalsamierung. Seine politische Sinndimension ist ihm wohl erst in der Spätzeit, in der Zeit der Fremdherrschaft, zugewachsen. Auch andere Rituale haben erst in der Spätzeit einen politischen Sinn angenommen, zum Beispiel das Ritual des Ziehens der vier Stoffkästen..$^{12}$ Dieses Ritual, in dem vier Kästen mit grünem, rotem, weißem

9 Beinlich (Anm. 6), S. 147; Cauville (Anm. 6), Bd.1, S. 46 (Dendara X, 82).

10 Beinlich (Anm. 6), S. 149; Cauville (Anm. 6), Bd.1, S. 46 (Dendara X, 82).

11 Beinlich (Anm. 6), S. 153; Cauville (Anm. 6), Bd.1, S. 46 (Dendara X, 83).

12 Vgl. A. Egberts: In Quest of Meaning. A Study of the Ancient Egyptian Rites of Consecrating the Meret-Chests and Driving the Calves. Leiden 1993. 
und jrtjw-Leinen zuerst ıgeweiht « und dann 'gezogen « werden, stammt ursprünglich aus dem Sokarfest, aus dem auch die Choiakmysterien hervorgegangen sind, und hängt daher eng mit dem Totenkult zusammen. Die vier Leinenstoffe dienen der Einbalsamierung und Beisetzung des Sokar-Osiris. ${ }^{13}$ In der ptolemäischen Zeit wird das Wort $m r t$ für `Kasten ‘ oft als t3-mrj (`Ägypten`) aufgefasst. Das >Weihen ` der Kästen wird jetzt gedeutet als das `Leiten ‘ (im Ägyptischen dasselbe Wort wie sweihen`) der Bewohner Ägyptens. Im begleitenden Spruch heißt es zum Beispiel: „Nimm Ägypten, indem es vereinigt ist. / Du hast die beiden Länder zu einem Ganzen verbunden. « Das Motiv der ,Verbindung deutet die >Binden aus, mit denen die Kästen umwunden sind. Das Wort »Ägypten« ( $(3-m r j)$ steht im Wortspiel mit dem Wort »Kasten« $(m r . t)$ :

Ich bringe dir Ägypten, indem es deiner Majestät zugeführt ist.

Das Land vergrößert den Schrecken vor dir. ${ }^{14}$

Der Ritus kann statt »die meret-Kästen ziehen für Amun« geradezu »Ägypten seinem Vater Amun zuführen " genannt werden..$^{15}$

Im Kontext des Osiris-Kults wird aber auch dieser Ritus auf die Glieder des Osiris hin ausgedeutet. Das Ziehen der vier Kästen bedeutet das Einsammeln der Glieder und ihre Vereinigung zum Osiris-Leib. ${ }^{16}$

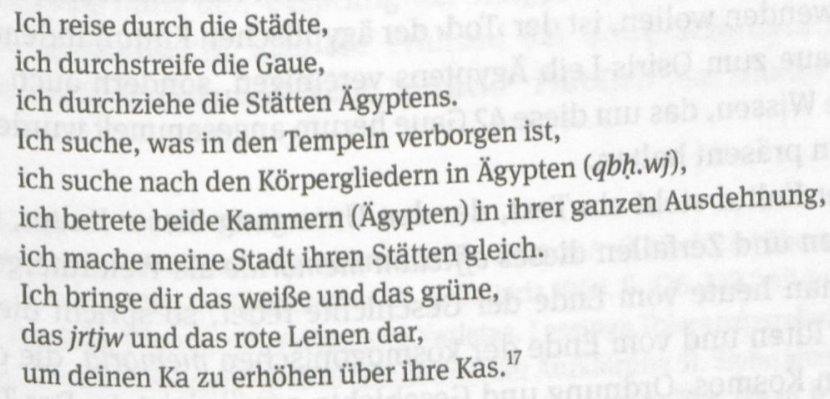

So bildeten die Ägypter den zergliederten Körper des Osiris auf die Vielheit der Gaue ab, um im Ritual der Gliedervereinigung die Einheit, Ganzheit und Unversehrtheit des Landes Ägypten zu begehen. Es scheint mir ein Irrtum zu meinen, dass es sich hier nur um eine Variante des verbreiteten Vegetationsmythos vom

\footnotetext{
13 Vgl. ebd., S. 173-199.

14 Ebd., S. 153 f. und 131.

15 Ebd., S. 96.

16 Vgl. ebd., S. 179.

17 Ebd., S. $153 \mathrm{f}$.
} 
sterbenden und auferstehenden Saatkorn handelt. Natürlich gehört auch dieses Motiv zum Urbestand des Osiris-Mythos. Aber es ist ganz sicher nicht die Sorge um das Keimen der Saat und die Wiederkehr der Vegetation, die diesen Mythos und seine Semantik des Zerreißens und Wiederzusammenfügens in den Mittelpunkt der spätägyptischen Kultur rückt. Dahinter steht vielmehr die Sorge um den Fortbestand dieser Kultur selbst, und zwar über eine Krise hinweg, die als Zerrissenheit gedeutet und im Kult nachvollzogen wird. In dér griechisch-römischen Zeit erscheint die ägyptische Kultur als ein Sinnzusammenhang, der in der Zeit der Fremdherrschaft und der hegemonialen Ausbreitung der hellenistischen Kultur immer stärker von Vergessen und Desintegration bedroht ist. So darf man auch nicht vergessen, dass Seth, der Mörder des Osiris und Zerstückeler seines Leichnams, in dieser Zeit immer stärker den Charakter des Asiaten, Eindringlings, Vandalen und Religionsfrevlers annimmt. Die mörderische Attacke des Seth erhält einen neuen politischen Sinn. Sie verkörpert die Gefahr, von der sich Ägypten permanent bedroht sieht und die immer im Norden lokalisiert wird. Das große Triumphfest des Horus von Edfu inszeniert den Mythos so, dass Seth von Norden in Ägypten einfällt und von Süden aus durch Horus zurückgeschlagen wird. Das Thema der sInvasion von Norden spielt auch in anderen Mythen der Spätzeit eine große Rolle. ${ }^{18}$ In der politischen sakramentalen Ausdeutung dieser Feste und Riten - und besonders der Choiakriten - artikuliert sich eine nationalägyptische Reaktion auf die persische, griechische und römische Fremdherrschaft. Was die Choiakriten abwenden wollen, ist der `Tod` der ägyptischen Kultur, indem sie nicht nur die 42 Gaue zum Osiris-Leib Ägyptens vereinigen, sondern auch das immense kulturelle Wissen, das um diese 42 Gaue herum angesammelt wurde, in Listen und Liturgien präsent halten.

Am Ende dieser Kultur steht ein Text, der den Untergang dieser Kultur, das endgültige Vergessen und Zerfallen dieses officium memoriae als Weltuntergang schildert. So wie man heute vom Ende der Geschichte redet, so spricht dieser Text vom Ende der Riten und vom Ende der kosmogonischen memoria, die den Zusammenhang von Kosmos, Ordnung und Geschichte gewährleistete. Der Text steht im Corpus Hermeticum:

Und doch wird eine Zeit kommen, wenn es so aussieht, als hätten die Ägypter vergeblich die Gottheit verehrt mit frommem Herzen und unablässiger Hingabe, und alle heilige Hinwendung zu den Göttern wird vergeblich und ihrer Früchte beraubt sein. Denn die Gottheit wird von der Erde wieder zum Himmel aufsteigen und Ägypten verlassen. Dieses Land, einst der Sitz der Religion, wird nun der göttlichen Gegenwart beraubt sein. Fremde werden

18 Vgl. Donald B. Redford: Pharaonic King-Lists, Annals and Day-Books. A Contribution to the Study of the Egyptian Sense of History. Mississauga 1986 (SSEA Publication 4), S. 277-281. 
dieses Land bevölkern, und die alten Kulte werden nicht nur vernachlässigt, sondern geradezu verboten werden. Von der ägyptischen Religion werden nur Fabeln übrig bleiben und beschriftete Steine. [...] In jenen Tagen werden die Menschen des Lebens überdrüssig sein und aufhören, den Kosmos (mundus) zu bewundern und zu verehren. [...]

Die Götter werden sich von den Menschen trennen - o schmerzliche Trennung! [...]

In jenen Zeiten wird die Erde nicht länger fest sein und das Meer nicht mehr schiffbar, der Himmel wird die Sterne nicht in ihren Umläufen halten noch werden die Sterne ihre Bahn im Himmel einhalten; jede göttliche Stimme wird notwendig zum Schweigen kommen. Die Früchte der Erde werden verfaulen, der Boden wird unfruchtbar werden und die Luft selbst wird stickig und schwer sein. Das ist das Greisenalter der Welt: das Fehlen von Religion (inreligio), Ordnung (inordinatio) und Sinn (inrationabilitas). ${ }^{19}$

Der Abbruch der Riten und das Ende der Kosmosverehrung versetzt die Welt in den Zustand akuter Todesbefallenheit. Nach altägyptischer Überzeugung sind die Riten für die Welt, was der Blutkreislauf für den Körper ist: Sie binden, verbinden, verknüpfen und beleben. Ihr Verfall lässt die Einheit der Welt zerfallen und gibt sie der Verwesung preis.

$\mathrm{Ob}$ es nun einen Zusammenhang gibt zwischen der einstweilen nur griechisch bezeugten Geschichte von der List der Isis und anderen, bisher auch nur unzureichend erforschten Vorstufen der Ringparabel in christlichen, islamischen oder (wie Friedrich Niewöhner vermutete ${ }^{20}$ ) in jüdischen Quellen und wie dieser Überlieferungsweg zu rekonstruieren wäre, bleibt eine offene Frage. Der neueste Vorschlag führt den Ursprung der Ringparabel auf eine christliche Quelle des 8./9. Jahrhunderts, also die Frühzeit der Religionsgespräche zwischen Christentum, Judentum und Islam, zurück. ${ }^{21}$ Plutarch und Diodor waren vielgelesene

19 Asclepius 24-26. In: Corpus Hermeticum. Hg. von A. D. Nock und übersetzt von A.-J. Festugière. Bd. 2: Traités XIII-XVIII. Asclepius. 2. Aufl. Paris 1960, S. 326-329 (mit Auslassungen).

20 Friedrich Niewöhner: Veritas sive Varietas. Lessings Toleranzparabel und das Buch Von den drei Betrügern. Heidelberg 1988 (Bibliothek der Aufklärung 5). Siehe jetzt auch Marcel Poorthuis: The Three Rings. Between Exclusivity and Tolerance. In: The Three Rings. Textual Studies in the Historical Trialogue of Judaism, Christianity and Islam. Hg. von Barbara Roggema, Marcel Poorthuis und Pim Valkenberg. Leuven, Dudley 2005 (Publications of the Thomas Instituut te Utrecht. New Series 11), S. 257-285, sowie Iris Shagrir: The Parable of the Three Rings. A Revision of its History. In: Journal of Medieval History 23 (1997), S.163-177.

21 Die bislang älteste bekannte Variante findet sich im Dialog des Patriarchen Timotheos und des Kalifen Al-Mahdi, um 781, nach Karl-Josef Kuschel: Im Ringen um den wahren Ring. Lessings "Nathan der Weise« - eine Herausforderung an die Religionen. Ostfildern 2011, 153f.; siehe hierzu Timotheos I., Ostsyrischer Patriarch: Disputation mit dem Kalifen Al-Mahdi. Hg. von Martin Heimgartner. 2Bde. Louvain 2011 (Corpus scriptorum Christianorum orientalium 631 und 632, Scriptores Syri 244 und 245). In dieser Fassung wirft jemand eine wertvolle Perle, die alle begehren, in ein finsteres Haus. Jeder glaubt, sie gefunden zu haben, aber erst wenn es hell wird, stellt sich die Wahrheit heraus. 
Autoren, deren Kenntnis bei christlichen, muslimischen und jüdischen Gelehrten des Mittelalters vorausgesetzt werden darf. Trotzdem bedeutet es doch einen sehr weiten Schritt von der Frage nach dem echten Osiris-Grab zu der Frage nach der wahren Religion. Weder bei Plutarch noch bei Diodor wird die Episode mit einer der Ringparabel vergleichbaren Botschaft verbunden, des Sinnes etwa: $\mathrm{Ihr}$ Gaue Ägyptens, vertragt euch und respektiert einander, denn euer Anspruch auf Wahrheit hat jeweils gleiche Berechtigung und keiner unter euch hat den anderen irgendetwas voraus. Tut eure Pflicht und ehrt das Grab, das Euch gegeben ist, ohne den anderen ihre entsprechenden Pflichten und Ansprüche streitig zu machen.

Eine solche Moral der Geschichte hätte aber immerhin Plutarchs Grundüberzeugung von der letztlichen Einheit aller Religionen entsprochen. An einer anderen Stelle seines Traktats über Isis und Osiris schreibt er, dass »ebenso wie die Sonne, der Mond, der Himmel, die Erde und das Meer allen gemeinsam sind, obwohl sie bei den verschiedenen Völkern mit verschiedenen Namen bezeichnet werden «, auch »die eine Vernunft (logos), die alles ordnet, und die eine Vorsehung, die für alles sorgt", bei den "verschiedenen Völkern mit verschiedenen Ehren, Anredeformen und geheiligten Symbolen « verehrt werden. ${ }^{22}$ Alle Völker verehren denselben Logos und dieselbe Vorsehung, nur in den ihnen eigentümlichen Riten, Sprachen und Symbolen, so wie auch alle denselben Kosmos kennen und nur mit den ihnen jeweils eigenen Begriffen bezeichnen.

Aus dieser Plutarch-Stelle spricht schon derselbe Geist des Kosmopolitismus, der auch Lessings Verwendung der Ringparabel kennzeichnet. Plutarch, Diodor und vermutlich schon Hekataios von Abdera, ein Hellenist der ersten Stunde, lebten und schrieben in einem Zeitalter der Globalisierung, in dem die verschiedenen Kulturen und Religionen einander nahegerückt waren und das Griechische, so wie heute das Englische, als ein Medium fungierte, sich einander verständlich zu machen und miteinander zu kommunizieren. Mag sein, dass auch das Motiv von der List der Isis, die Wahrheit zu verhüllen und auf alle zu verteilen, von diesem kosmopolitischen Geist geprägt ist. Das spätägyptische Ritual aber, das dahintersteht und die griechischen Autoren vermutlich in ihrem Sinne missverstanden haben, atmet einen ganz anderen Geist: die Angst vor dem Verlust der politischen, religiösen und kulturellen Identität in den Jahrhunderten der Fremdherrschaft und des vom Hellenismus ausgehenden kulturellen Assimilationsdrucks. Diese Einheit wird als ein Leib imaginiert, der aus den Gliedern, in die er zu zerfallen droht, immer wieder rituell zusammengesetzt und beschworen werden muss. Ägypten als der Leib eines getöteten und verklärten Gottes - das lässt eher an Paulus denken und sein Konzept der Kirche als Leib Christi, aber kaum an die Ringparabel.

22 Plutarch: De Iside et Osiride 67 (377 E-F). 